\title{
Wireless Sensor Network for Continuous Monitoring a Patient's Physiological Conditions Using ZigBee
}

\author{
Ramanathan.P \\ ECE-DEPT Pallavan College of Engineering \\ Thimmasamudram, Kanchipuram-631502 \\ Tamilnadu, India \\ Tel: 91-094-4553-7924Ｅ-mail: dr.ramanathan.pad@gmail.com \\ Pradip Manjrekar.P \\ Flat No.248, CGS colony, near wadala R.S.post office \\ Wadala (west), Mumbai-400 031, India \\ Tel: 91-095-70348898 E-mail: dr.pradippm1@gmail.com
}

Received: March 31, 2011

Accepted: July 29, 2011

doi:10.5539/cis.v4n5p104

\begin{abstract}
The research paper, presents a Wireless Sensor Network (WSN) for monitoring a patient's physiological conditions continuously using Zigbee. In the experiment 3 groups of persons were made to undergo the test. Person A was in the age group of 1-10 years. Person B was in the age group of 11-30 years and person C was in the age group of 31-80 years. Physiological conditions of these 3 groups of persons were monitored using physiological sensor. The output of physiological sensor has to be transmitted via Zigbee and the same has to be sent to the remote wireless monitor for acquiring the observed patient's physiological signal. The remote wireless monitor is constructed of Zigbee and Personal Computer (PC). The measured signal has to be sent to the PC, which can be data collection. When the measured signals over the standard value, the personal computer sends Global System for Mobile communication (GSM) short message to the care taker.

Although Bluetooth is better than Zigbee for transmission rate, Zigbee has lower power consumption. Hence, Zigbee is generally used for 24 hours monitor of communication transmission system. The first procedure of the system is that Physiological sensor is used to measure Heart rate, respiration rate, pulse rate, temperature, oxygen level in the blood and blood pressure from human body using Zigbee. Next procedure of the system is to measure saline level in bottle using zigbee.The measured signal is to be sent to the PC via the RS-232 serial port communication interface. The signal is to be sent to remote PC or Personal Digital Assistant (PDA) from the internet. In particular, when measured signals over the standard value, the personal computer will send GSM short message to absent care taker's mobile phone. The results obtained using WSN in terms of accuracy and credibility of measurement is poor. But, the result obtained from WSN using zigbee confirms that it effectively works for continues monitoring a patient's physiological conditions. The conclusion and implications of this study reveals that a successful research project was developed and the abnormal conditions of the patients were identified. So the effectiveness of this WSN using zigbee is more compared with the effectiveness of WSN.
\end{abstract}

Keywords: Physiological-sensor, Zigbee, Relays, Voice Board, Low voltage power amplifier, Power supply, RS232 serial communication, Max 232, Timers, ADC module and computer

\section{Introduction}

In the current Hi Tech, wireless communication place the major role which led us the innovative idea of using this new technology for many applications. A Wireless Sensor Network (WSN) is a wireless network consisting of spatially distributed autonomous devices using sensors to monitor physical or environmental conditions.

After around a decade of active research on wireless sensor network recent standards released are stimulating the development of commercial products. One of standards is zigbee. This paper highlights some of the lessons from the field that went into the standard. It also describes the ecosystems emerging around zigbee and the enabling trends in that ecosystem. New area of activity in the zigbee standards body is highlighted as well (Safaric 
S.Malaric K, 2006). The development of wireless sensor networks was originally motivated by military application such as battlefield surveillance.

The joint efforts of the IEEE 802.15.4 task group and the zigbee Alliance have ended up with the specification of a standard protocol stack for Low-Rate Wireless Personal Area Networks (LR-WPANs), a promising technology for Wireless Sensor Networks (WSNs) (J.zheng \& J.L Myung, 2004) (D.Geer, 2005) (J.Adams, 2005) (T. Culter, 2005) (C.Herzog, 2005). In what follows, we denote by Zigbee the entire IEEE 802 15.4/Zigbee protocol stack.

Zigbee is gaining an exponentially increasing interest from industry and is considered as a universal solution for low-cost low-power wirelessly connected monitoring and control devices(J.Adams, 2005) (T. Culter, 2005) (C.Herzog, 2005). This interest is mainly driven by the potentially large number of emerging applications including home automation (as the current principal commercial target of the Zigbee Alliance), health care monitoring, industrial automation, environmental monitoring, surveillance and so on, These applications have essentially been triggered by the wireless sensor network praigm, which represents the new generation of network infrastructure for large-scale distributed embedded systems.

The research was done to apply this WSN using zigbee for healthcare system.(Ze Zhao \& Li Cui, 2005) the objective of this research project is to study the effectiveness of WSN using zigbee and to identify the abnormal conditions of these patients.

First a proper sensor has to be chosen for converting the physiological signal into electrical signal in the form of analog signal. This analog signal has to be converted into digital signal by designing a proper circuit. This digital signal is fed into the Peripheral Interface Controller (PIC) and its subsequent output is connected to the serial communication circuit. The output of this serial communication circuit is fed into the zigbee device and output of this zigbee device is transmitted via transmitting antenna. In the receiver side the said transmitted signal is received through the receiving antenna and fed into the zigbee unit. The output of this zigbee unit is fed into the RS-232 serial port communication interface and output of this RS-232 is fed into Personal Computer (PC) sends Global System for Mobile communication (GSM) short message to the receiver. The receiver can use the PC or Personal Digital Assistant (PDA) to observe the sensed signals in the remote place.

The PIC microcontrollers are supported with a full range of hardware and software development tools (David Gay, et al, 2003). The transmission section codes using various softwares to operate the transmission of sensed digital signals were found. Also, softwares were found to operate the received signals.

\section{Methodology}

The name Zigbee is said to come from the domestic honey bee which uses a zigbee of dance to communicate important information to other members. This communication dance is what engineers are trying to emulate with this protocol a bunch of separate and simple organisms that join together to take complex tasks. Zigbee is provided with lower consumption, small volume, high expansion, stylization and two way transmission, etc.

The Zigbee protocol is implemented on top of the IEEE 802.15.4 radio communication standard. The Zigbee specification is managed by a non-profit industry consortium of semiconductor manufacturers, technology providers and other companies, all together designated the Zigbee alliance. The alliance currently numbers more than 150 members.

The Zigbee specification is designed to utilize the features supported by IEEE 802.15.4. In particular, the scope of Zigbee is applications with low requirements for data transmission rates and devices with constrained energy sources.

The intended market spaces for Zigbee products include home control and building automation. Imagine the intelligent building, controlling the lighting and temperature as needed, monitoring the building structure and performing surveillance tasks with a minimum of user interaction. This is the potential of Zigbee. (Figure 1)

Two message types are defined:

1. Key value pair (KVP) service which uses a standardized way of representing messages using binary XML.

2. Message (MSG) service which gives full control over the messages being sent for application specific needs.

\subsection{Comparison of wireless technologies}

The comparison of wireless technologies is shown in Table 1. 


\subsection{Data Flow Diagram:}

The flow diagram is shown in Figure 2. The Zigbee/Zigbee-PRO OEM RF Modules interface to a host device through a logic-level asynchronous serial port. Through its serial port, the module can communicate with any logic and voltage compatible UART; or through a level translator to any serial.

Today, organizations use Zigbee to effectively deliver solutions for a variety of areas including consumer electronic device control, energy management and efficiency, home and commercial building automation as well as industrial plant management. With this comprehensive set of attributes, the nonprofit, open membership and volunteer driven alliance has become a thriving ecosystem of more than 300 members. As an ecosystem, the alliance offers everything prospective product and service companies need to develop Zigbee products and services and benefit from the alliance's competitive and stable supply chain.

The health care domain presents opportunities for a significant number of applications of wireless sensor technology. The following sections focus on broad health monitoring applications that include chronic disease monitoring, personal wellness monitoring and personal fitness. Within each of these applications, we describe several specific uses of wireless sensor technology. Firstly, pulse rate measurements for the patients A, B \& C were taken from their forehand. Secondly, using heart sensor, heart rates for the patients A, B \& C were taken. Next, using the micro $\log$ external temperature sensor temperature from the body of patients $\mathrm{A}, \mathrm{B} \& \mathrm{C}$ were taken. Next patients A, B \& C were given saline. When the bottle becomes empty the ray passing through the bottle gets destructed and the ray is cut. So, the LED glows and this indicates that the bottle became empty.Next, blood pressure of patients A, B \& C were taken. The skin may be illuminated with visible (red) or infrared LEDs using transmitted or reflected light for detection. The very small changes in reflectivity or in transmittance caused by the varying blood content of human tissue are almost invisible. Finally, to find oxygen level in the blood, patients A, B $\& \mathrm{C}$ were tested for $\mathrm{SO} 2$ level. As shown in Figure 3 and Figure 4 firstly appropriate sensor was chosen for the above applications to convert the physiological signal into electrical signal which is in the form of analog signal. This analog signal was converted into digital Signal by designing a proper circuit. This digital signal was fed into the PIC controller. The output of this PIC controller is fed into the serial communication circuit. The output of this serial communication circuit is fed into the Zigbee device and output of this Zigbee device is transmitted via transmitting antenna.

As shown in the under mentioned Figure 5 in the receiver side the said transmitted signal is received through the receiving antenna and fed into the Zigbee unit. The output of this Zigbee unit is fed into the RS-232 serial port communication interface and output of this RS-232 is fed into Personal Computer (PC) which can be data collection. This Personal Computer (PC) sends Global System for Mobile communication (GSM) short message to the receiver. The receiver can use the PC or Personal Digital Assistant (PDA) to observe the sensed signals in the remote place.

\section{Result Analysis}

- Firstly, pulse rate measurements for the patients A, B and C were taken.. When abnormality is observed and the pulse drop is below the predetermined level then a call is dialed to the doctor, near by hospital and to the residence indicating the critical condition of the patient. Hence, the numbers were dialed successfully to the doctor, near by hospital and to the residence. This indicates the success of the experiment. Also, sensed signals were observed through Personal Computer (PC) or Personal Digital Assistant (PDA). This indicates the success of the experiment..

- Secondly, heart rate for patients A, B and C were taken.. When the heart rate crosses 72 beats per second and when it is lower than 72 beats per second, the condition was identified as abnormal. Hence, in such a situation automatically a call is dialed to the doctor, nearby hospital and to the residence. Hence, the research is considered successful. Also sensed signals were observed through Personal Computer (PC) or Personal Digital Assistant (PDA).Hence, this experiment is considered successful.

- Thirdly, patients A, B and C were tested for respiration rate measurement. Respiration rates above the normal level or below the normal level (when at rest) may be considered abnormal. In such a situation automatically a call is dialed to the doctor, nearby hospital and to the residence. Also, sensed signals were observed through Personal Computer (PC) or Personal Digital Assistant (PDA). Therefore, research is considered successful.

- Fourthly, temperature measurements for the patients A, B and C were taken. While the temperature goes below the normal condition or above the normal condition, LED (yellow) glows and automatically a call is dialed to the doctor, nearby hospital and to the residence who have given their phone or cell number indicating that the 
patient is in abnormal condition. Hence, the research is successful. Also sensed signals were observed through Personal Computer (PC) or Personal Digital Assistant (PDA). Hence, research is considered successful.

Patients A, B and C were given saline. When the bottle becomes empty, the ray passing through the bottle gets destructed and the ray is cut. So, the LED glows. Now automatically call is dialed to the doctor, nearby hospital and to the residence. Hence the experiment is considered successful.

To find oxygen level in the blood, patients $\mathrm{A}, \mathrm{B}$ and $\mathrm{C}$ were tested for $\mathrm{SaO}_{2}$ level. When the ratio goes low, a call was automatically dialed to the doctor, nearby hospital and to the residence. Hence the experiment is considered successful.

\section{Conclusion}

This work represents an important step in understanding the complexity of the deployment of cluster-tree topologies in IEEE 802.15.4/zigbee WAPNs and paves the way for their deployment. We are currently working on the real implementation of these proposals in our experimental test bed, which already implementation of these proposals in our experimental test bed, which already implements the basic features of the IEEE 802.15.4/zigbee protocol stack build on top of MICAz motes from Crossbow.

The above results prove that the objective of the research was achieved successfully.

\section{References}

(2003). IEEE-TG15.4 part 15.4: wireless Medium Access Control (MAC) and physical Layer (PHY) Specifications for Low-Rate Wireless Personal Area Networks (LR-WAPNs). IEEE standard for information Technology.

C.Herzog. (2005). Creating value with zigbee networks. Industrial Embedded System Resource Guide, Networking: Technology, pp. 31-33.

D. Geer. (2005). Users make a Beeline for zigbee Technology. IEEE computer society press, 38(12), pp.16-19.

David Gay, Philip Levis, David Culler \& Eric Brewer. (2003). NesC1.1 Language Reference Manual.

J.Adams. (2005). Building low power into wireless sensor networks using zigbee technology. Industrial Embedded systems Resources Guide, Networking: Technology, pp 26-30.

J.zheng \& J.L Myung. (2004).Will IEEE 802.15.4 make Ubiquitous Networking a reality? — A Discussion on a Potential Low Power Low Bit Rate Standard. IEEE Communications Magazine, 42(6), pp.140-146.

Safaric S.Malaric K. (2006). ZigBee wireless standard. Multimedia signal processing and communications, $48^{\text {th }}$ international symposium ELMAR 2006, Zadar, Croatia.

T. Culter. (2005). Deploying Zigbee in exiting industrial automation networks. Industrial Embedded Systems Resource Guide, Networking: Technology, pp.34-36.

Ze Zhao \& Li Cui. (2005). EasiMed: Aremote health care solution. Proceeding of the 2005 IEEE Engineering in medicine and Biology $27^{\text {th }}$ annual conference, Shanghai, China.

Zigbee-Alliance. Zigbee specification. http:// [Online] Available: zigbee.org/2005. 
Table 1. Comparison of wireless technologies

\begin{tabular}{|l|l|l|l|}
\hline & Zigbee & Bluetooth & Wi-Fi \\
\hline Standard & 802.15 .4 & 802.15 .1 & $802.11 \mathrm{~b}$ \\
Memory & $4-32 \mathrm{~KB}$ & $250 \mathrm{~KB}+$ & $1 \mathrm{MB}+$ \\
Battery & Years & Days & Hours \\
Nodes per master & $250 \mathrm{~Kb} / \mathrm{s}$ & $1 \mathrm{Mb} / \mathrm{s}$ & $11 \mathrm{Mb} / \mathrm{s}$ \\
Data rate Range & $300 \mathrm{~m}$ & $10 \mathrm{~m}$ & $100 \mathrm{~m}$ \\
\hline
\end{tabular}

\begin{tabular}{|c|}
\hline $\begin{array}{c}\text { Application Framework } \\
\text { (AF) }\end{array}$ \\
\hline $\begin{array}{c}\text { Application Support Sub layer } \\
\text { (APS) }\end{array}$ \\
\hline Network Layer(NWK) \\
\hline Medium Access Control \\
Layer(MAC)
\end{tabular}

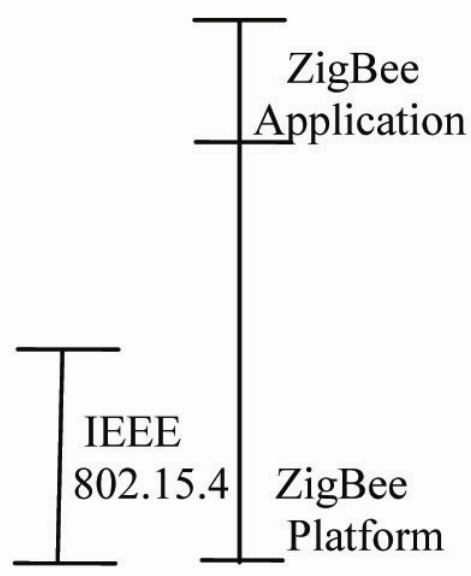

Figure 1. ZigBee Protocol supported by IEEE 80215.4 


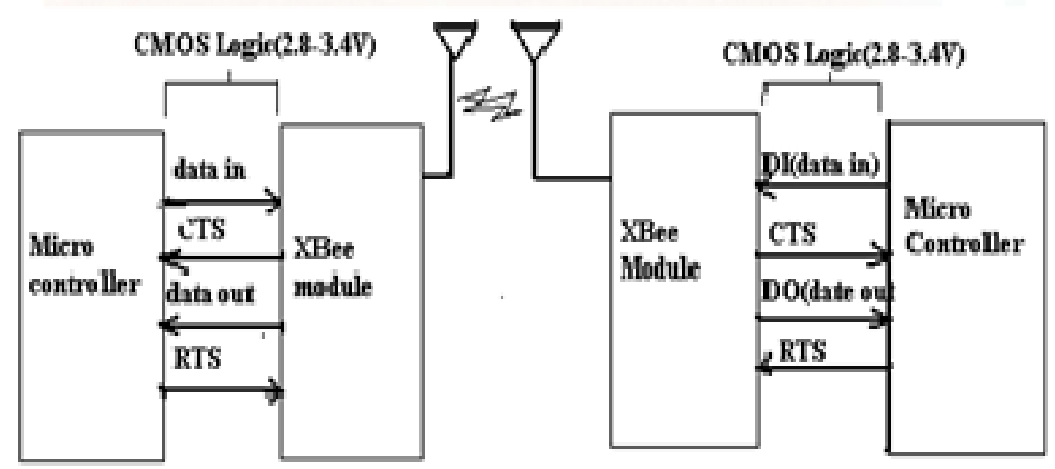

Figure 2. Data Flow Diagram

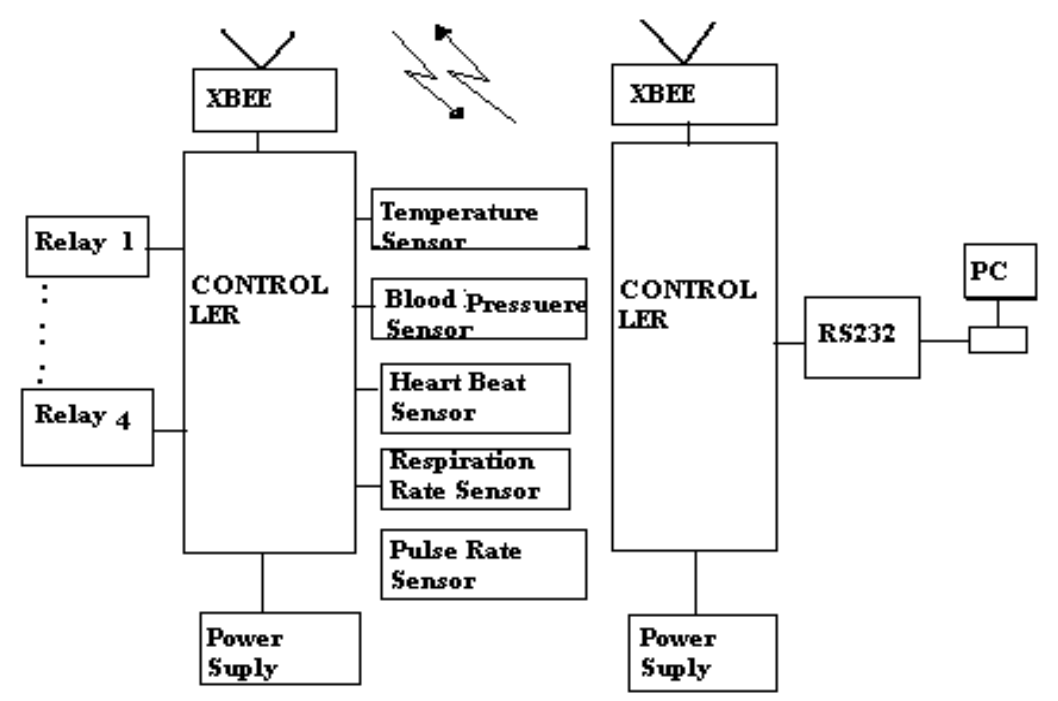

Figure 3. Block Diagram of Transmitter Section \& receicer

using Zigbee

PATIENT SECTION:

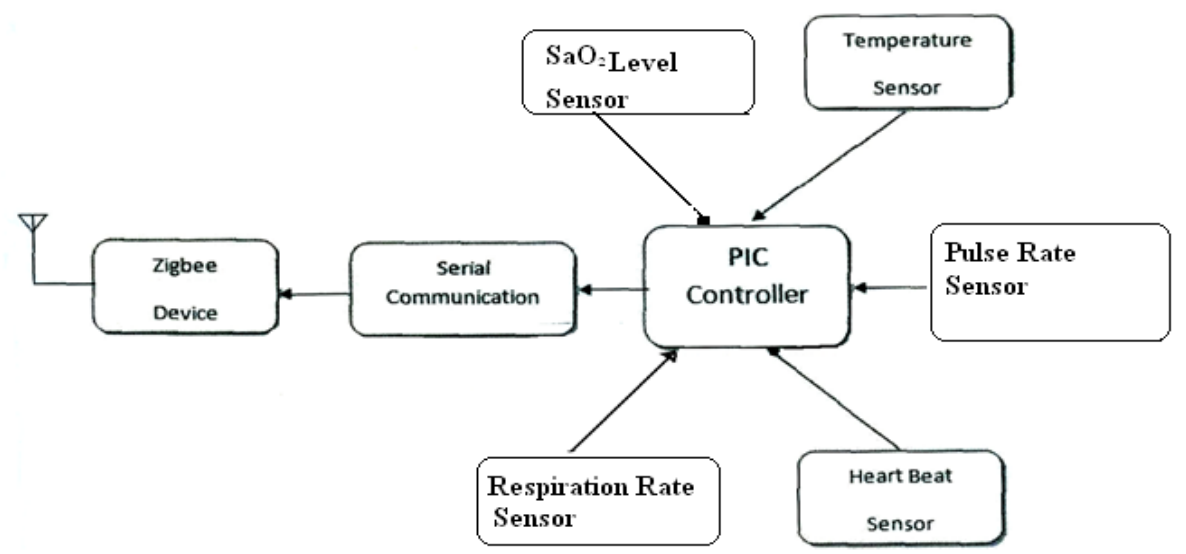

Figure 4. Block Diagram of Transmitter Section 


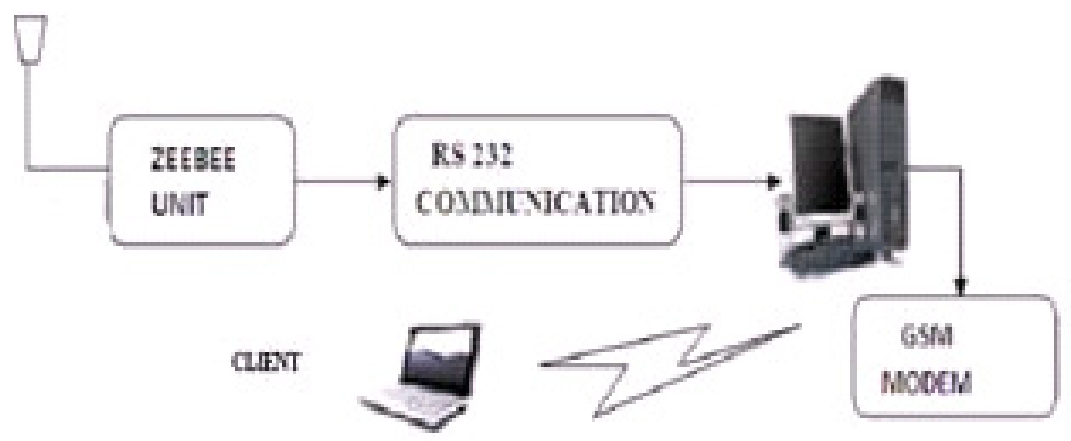

Figure 5. Block Diagram of Receiver Section 